\title{
QUEEN'S
UNIVERSITY
BELFAST
}

\section{A Measurements Based Comparison of New and Classical Models Used to Characterize Fading in Body Area Networks}

Cotton, S. L., Yoo, S. K., \& Scanlon, W. G. (2014). A Measurements Based Comparison of New and Classical Models Used to Characterize Fading in Body Area Networks. In 2014 IEEE MTT-S International Microwave Workshop Series on RF and Wireless Technologies for Biomedical and Healthcare Applications (IMWS-Bio 2014) Institute of Electrical and Electronics Engineers Inc.. https://doi.org/10.1109/IMWS-BIO.2014.7032411

Published in:

2014 IEEE MTT-S International Microwave Workshop Series on RF and Wireless Technologies for Biomedical and Healthcare Applications (IMWS-Bio 2014)

\section{Document Version:}

Peer reviewed version

Queen's University Belfast - Research Portal:

Link to publication record in Queen's University Belfast Research Portal

\section{Publisher rights}

( $) 2015$ IEEE. Personal use of this material is permitted. Permission from IEEE must be obtained for all other uses, in any current or future media, including reprinting/republishing this material for advertising or promotional purposes, creating new collective works, for resale or redistribution to servers or lists, or reuse of any copyrighted component of this work in other works.

\section{General rights}

Copyright for the publications made accessible via the Queen's University Belfast Research Portal is retained by the author(s) and / or other copyright owners and it is a condition of accessing these publications that users recognise and abide by the legal requirements associated with these rights.

Take down policy

The Research Portal is Queen's institutional repository that provides access to Queen's research output. Every effort has been made to ensure that content in the Research Portal does not infringe any person's rights, or applicable UK laws. If you discover content in the Research Portal that you believe breaches copyright or violates any law, please contact openaccess@qub.ac.uk. 


\title{
A Measurements Based Comparison of New and Classical Models Used to Characterize Fading in Body Area Networks
}

\author{
Simon L. Cotton, Seong Ki Yoo and William G. Scanlon \\ Institute of Electronics, Communications and Information Technology \\ Queens University Belfast, Queens Island, Belfast BT3 9DT, United Kingdom \\ Email: \{simon.cotton, s.yoo02,w.scanlon\}@qub.ac.uk
}

\begin{abstract}
In this paper we compare a number of the classical models used to characterize fading in body area networks (BANs) with the recently proposed shadowed $\kappa-\mu$ fading model. In particular, we focus on BAN channels which are considered to be susceptible to shadowing by the human body. The measurements considered in this study were conducted at 2.45 GHz for hypothetical BAN channels operating in both anechoic and highly reverberant environments while the person was moving. Compared to the Rice, Nakagami and lognormal fading models, it was found that the recently proposed shadowed $\boldsymbol{\kappa}-\boldsymbol{\mu}$ fading model provided an enhanced fit to the measured data.

Index Terms - Body centric communications, on-body fading channels, channel characterization.
\end{abstract}

\section{INTRODUCTION}

A full understanding of the fading characteristics likely to be encountered by body area networks (BANs) is essential for the design of robust pervasive medical applications. One of the most dominant factors observed in on-body fading channels used to interlink wireless nodes in BANs is body shadowing [1]. Body shadowing occurs when one or more parts of the human body obscure the direct line of sight (LOS) signal path between the transmitter and receiver. In on-body communication channels, shadowing caused by body parts obstructing the direct link between antennas can cause substantial attenuation of the received signal. Body shadowing has previously been observed in on-body channel measurements [1, 2] and simulations [3] conducted at $2.45 \mathrm{GHz}$. Moreover, it is postulated that body shadowing will make future non-LOS (NLOS) millimeter-wave on-body communications at $60 \mathrm{GHz}$ [4] very difficult, if not impossible.

A new statistical fading model for shadowed body centric communications channels was recently proposed in [1]. In this model, clusters of multipath are assumed to have scattered waves with identical powers, alongside the presence of elective dominant signal components - a scenario which is identical to that observed in $\kappa-\mu$ fading [5]. The difference between the model proposed in [1] and that of $\kappa-\mu$ fading is that the resultant dominant component, formed by phasor addition of the individual dominant components is assumed to be a log-normally distributed random variable. In this paper, we characterize the on-body channels presented in [1] using an alternative fading model, namely the shadowed $\kappa-\mu$ fading model, in which the lognormal distribution of the resultant dominant component is superseded by the Nakagami- $m$ distribution. This method of modeling shadowed fading has recently and independently been proposed in [6] and [7]. Unlike that presented in [1], this model has the attractive feature that its probability density function (PDF) can be expressed in closed-form. To further demonstrate the utility of the shadowed $\kappa-\mu$ fading model for characterizing fading in BAN channels, we also compare its fit to field measurements with those obtained using classical fading models such as Rice [8], Nakagami [9] and lognormal [10] which are often used to model fading in BAN channels.

\section{MEASUREMENT SETUP AND EXPERIMENTS}

The measurements were conducted at $2.45 \mathrm{GHz}$ in the anechoic and reverberation chamber facilities at Queen's University Belfast, United Kingdom. The antennas used in this study were compact ( $5 \mathrm{~mm}$ height) higher mode microstrip patch antennas designed specifically for on-body networking applications. The test subject was an adult male of height $1.82 \mathrm{~m}$ and mass $90 \mathrm{~kg}$. In the experiments, the antennas were mounted so that the radiating patch element was parallel to the body surface. They were then connected to port 1 and 2 of a Rhode \& Schwarz ZVB-8 vector network analyzer (VNA) using calibrated low-loss coaxial cables. The VNA was configured with an output power of $0 \mathrm{dBm}$ and set to record measurements of $S_{21}$ at $5 \mathrm{~ms}$ intervals for 30 seconds. For all of the measurements presented here, the user performed a simulated walking motion at a set location in both environments. More details of the measurement setup and experiments can be found in [1] and the references therein.

\section{STATISTICAL CHARACTERIZATION}

A statistical characterization of the three on-body channels utilized in [1] namely the front-left-waist to the right-head, right-knee and right-wrist was performed using the shadowed $\kappa-\mu$, Rice, Nakagami and lognormal fading models. As the models associated with the Rice, Nakagami and lognormal fading channels are well known, we will focus on describing the shadowed $\kappa-\mu$ fading model. The PDF of the shadowed $\kappa-$ $\mu$ fading signal envelope, $R$, is given by 
TABLE I

PARAMETER ESTIMATES FOR EACH OF THE FADING MODELS FITTED TO THE MEASUREMENT DATA FROM THE ANECHOIC

CHAMBER. FOR THE RICE PDF, $A$ IS THE NON-CENTRALITY PARAMETER AND $s$ IS THE SCALE PARAMETER, FOR THE

NAKAGAMI PDF, $v$ IS THE SHAPE PARAMETER AND $\omega$ IS THE MEAN POWER AND FOR THE LOGNORMAL PDF, $\alpha$ IS THE MEAN AND $\beta$ IS THE STANDARD DEVIATION OF THE FADING SIGNAL'S LOGARITHM.

\begin{tabular}{|c|c|c|c|c|}
\hline On-Body Fading Channel & Shadowed $\boldsymbol{\kappa}-\mu$ & Rice & Nakagami & lognormal \\
\hline Left-waist to Right-Head & $\begin{array}{c}K=1.94, \mu=0.64, \hat{r}=1.01 \\
m=650, \Omega=0.60\end{array}$ & $\begin{aligned} A & =0.04 \\
s & =0.71\end{aligned}$ & $\begin{array}{l}v=0.85 \\
\omega=1.00\end{array}$ & $\begin{array}{c}\alpha=-0.35 \\
\beta=0.73\end{array}$ \\
\hline Left-waist to Right-Knee & $\begin{array}{c}K=1.36, \mu=0.88, \hat{r}=1.18 \\
m=948, \Omega=0.47\end{array}$ & $\begin{aligned} A & =0.63 \\
s & =0.55\end{aligned}$ & $\begin{array}{l}v=1.11 \\
\omega=1.00\end{array}$ & $\begin{array}{c}\alpha=-0.26 \\
\beta=0.59\end{array}$ \\
\hline Left-waist to Right-Wrist & $\begin{array}{c}\kappa=3.95, \mu=1.38, \quad \hat{r}=0.87 \\
m=0.39, \Omega=1.02\end{array}$ & $\begin{aligned} A & =0.03 \\
S & =0.71\end{aligned}$ & $\begin{array}{l}v=0.75 \\
\omega=1.00\end{array}$ & $\begin{array}{c}\alpha=-0.40 \\
\beta=0.71\end{array}$ \\
\hline
\end{tabular}

$$
\begin{aligned}
f_{R}(r)= & \frac{2 r^{2 \mu-1}}{\Gamma(\mu)}\left(\frac{\mu(1+\kappa)}{\hat{r}^{2}}\right)^{\mu} \\
& \times\left(\frac{m \hat{r}^{2}}{\mu(1+\kappa) \Omega+m \hat{r}^{2}}\right)^{m} \exp \left(-\frac{\mu(1+\kappa) r^{2}}{\hat{r}^{2}}\right) \\
& \times_{1} F_{1}\left(m ; \mu ; \frac{\Omega(\mu(1+\kappa) r)^{2}}{\hat{r}^{2}\left(\mu(1+\kappa) \Omega+m \hat{r}^{2}\right)}\right)
\end{aligned}
$$

where $\kappa$ is related to $\delta, \sigma$ and $\mu$ through the relationship $\kappa=\delta^{2} / 2 \mu \sigma^{2}$, which is simply the ratio of the total power of the dominant components $\left(\delta^{2}\right)$ to the total power of the scattered waves $\left(2 \mu \sigma^{2}\right)$ where $\mu$ is related to the multipath clustering and the mean power is given by $\hat{r}^{2}$. In (1), $\Gamma(\bullet)$ is the gamma function, ${ }_{1} F_{1}(\bullet ; \bullet ; \bullet)$ is the confluent hypergeometric function, $m=E^{2}\left[\Delta^{2}\right] / \operatorname{var}\left[\Delta^{2}\right]$ is the Nakagami parameter where $\operatorname{var}\left[\Delta^{2}\right]$ is the variance. In this instance, $\Omega=E\left[\Delta^{2}\right]$ is the average power of the resultant dominant component.

\section{RESULTS}

For convenience, the $r m s$ signal level, $\hat{r}=\sqrt{E\left[R^{2}\right]}$, was removed from the fading envelopes presented here to enable a direct comparison of the fading characteristics for each of the on-body links. All parameter estimates for the PDF of the shadowed $\kappa-\mu$ fading model were obtained using a non-linear optimization algorithm in MATLAB. Figures 1 and 2 show the empirical PDFs for the left-waist to right-knee and leftwaist to right-wrist on-body fading channels while the test subject was mobile in the anechoic chamber. Also shown for comparison are the PDFs of the shadowed $\kappa-\mu$, Rice, Nakagami and lognormal fading models. Visual inspection of Fig. 1 shows that for the right-knee positioned receiver, the shadowed $\kappa-\mu$ PDF provides the best fit. In this case, the Rice and Nakagami PDFs also provide a reasonable fit. This is in direct contrast to the right-wrist positioned receiver (Fig. 2), where the PDFs of the models associated with shadowed fading, i.e., the shadowed $\kappa-\mu$ and lognormal, were found to provide the best fit.

In this environment, multipath signals returned in the direction of the human body from the local surroundings will be minimised and hence the signal characteristics will be determined mostly by processes related to the body itself. This can be seen from Table I by observing the parameter estimates for the right-head and right-knee positioned antennas where $\mu<1$ and $\kappa>1$ suggesting that these channels suffer less from multipath and that a dominant component exists. As expected, the right-wrist positioned antenna exhibited the greatest amount of variation in the resultant dominant component $(m=0.39)$. This may be attributed to the body shadowing caused by the oscillatory movement of the arm from the front to the back of the body.

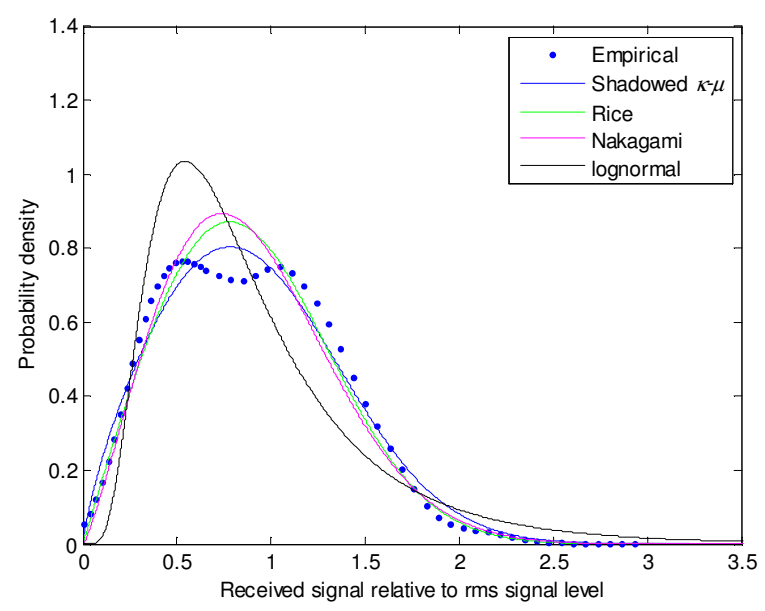

Fig. 1 Empirical and theoretical PDFs for right-knee positioned antenna in the anechoic chamber. 
TABLE II

PARAMETER ESTIMATES FOR EACH OF THE FADING MODELS FITTED TO THE MEASUREMENT DATA FROM THE REVERBERATION CHAMBER.

\begin{tabular}{|c|c|c|c|c|}
\hline On-Body Fading Channel & Shadowed $\kappa-\mu$ & Rice & Nakagami & lognormal \\
\hline Left-waist to Right-Head & $\begin{array}{c}\kappa=0.96, \mu=0.87, \hat{r}=1.04 \\
m=445, \Omega=0.49\end{array}$ & $\begin{aligned} A & =0.59 \\
s & =0.57\end{aligned}$ & $\begin{array}{l}v=1.02 \\
\omega=1.00\end{array}$ & $\begin{array}{c}\alpha=-0.28 \\
\beta=0.66\end{array}$ \\
\hline Left-waist to Right-Knee & $\begin{array}{c}\kappa=1.02, \mu=1.39, \hat{r}=1.13 \\
m=0.36, \Omega=0.42\end{array}$ & $\begin{aligned} A & =0.66 \\
s & =0.53\end{aligned}$ & $\begin{array}{l}v=1.24 \\
\omega=1.00\end{array}$ & $\begin{array}{c}\alpha=-0.23 \\
\beta=0.54\end{array}$ \\
\hline Left-waist to Right-Wrist & $\begin{array}{c}\kappa=12.6, \mu=0.79, \quad \hat{r}=1.04, \\
m=0.98, \Omega=0.88\end{array}$ & $\begin{aligned} A & =0.04 \\
S & =0.71\end{aligned}$ & $\begin{array}{l}v=0.88 \\
\omega=1.00\end{array}$ & $\begin{array}{c}\alpha=-0.33 \\
\beta=0.70\end{array}$ \\
\hline
\end{tabular}

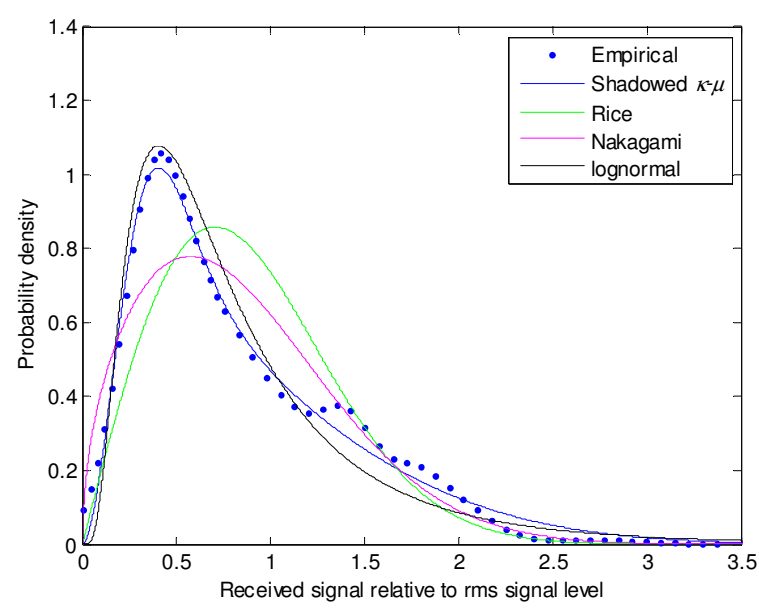

Fig. 2 Empirical and theoretical PDFs for right-wrist positioned antenna in the anechoic chamber.

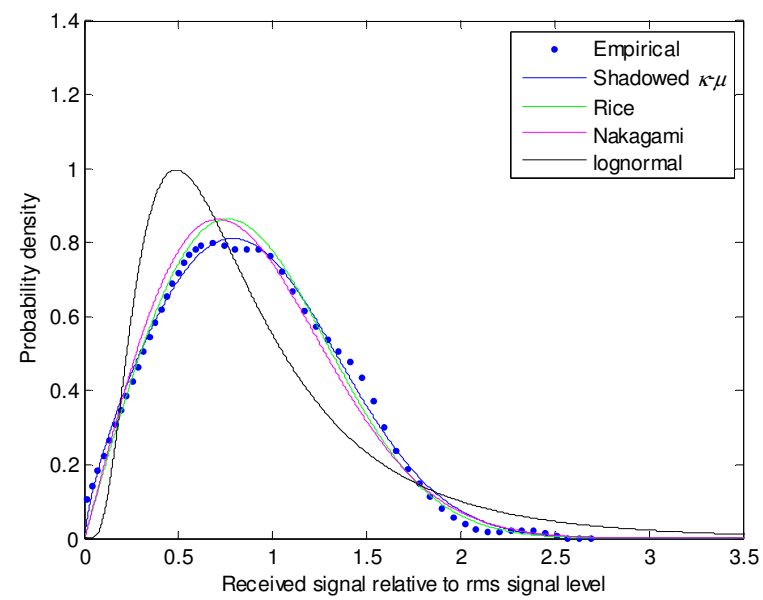

Fig. 3 Empirical and theoretical PDFs for the right-head positioned antenna in the reverberation chamber.

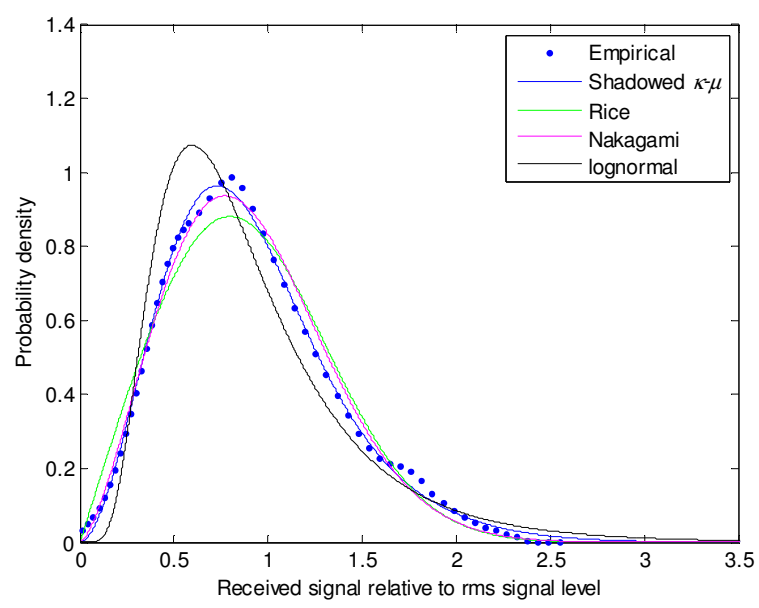

Fig. 4 Empirical and theoretical PDFs for right-knee positioned antenna in the reverberation chamber.

Figures 3 and 4 show the empirical PDFs for the left-waist to right-head and left-waist to right-knee on-body fading channels while the test subject was mobile in the reverberation chamber. Again we can see that the PDF of the shadowed $\kappa-\mu$ fading model provides the best fit. For both of these on-body fading channels, the Rice and Nakagami PDFs outperform the lognormal PDF showing that, as expected, these channels are more prone to multipath effects. This can be seen from Table II where, with the exception of the right-wrist positioned receiver, the estimated $\kappa$ parameters are reduced compared to the equivalent results for the anechoic chamber.

\section{CONCLUSION}

A number of statistical distributions used to model fading in BAN channels have been compared with measured data. It has been shown that the PDF of the recently proposed shadowed $\kappa-\mu$ fading model can provide an improved fit to channels which may be prone to shadowed fading compared to classical models such as Rice, Nakagami and lognormal. 


\section{ACKNOWLEDGEMENT}

This work was supported by the U.K. Royal Academy of Engineering and the Engineering and Physical Research Council (EPSRC) under Grant Reference EP/H044191/1 and by the Leverhulme Trust, UK through a Philip Leverhulme Prize.

\section{REFERENCES}

[1] S. L. Cotton, "A statistical model for shadowed body centric communications channels: theory and validation," IEEE Transactions on Antennas and Propagation, vol. 62, no. 3, pp. 1416-1424, March 2014.

[2] R. Rosini and R. D'Errico, "Comparing on-body dynamic channels for two antenna designs," Loughborough Antennas and Propagation Conference (LAPC), pp. 1-4, November 2012.

[3] C. Oliveira and L. M. Correia, "A statistical model to characterize user influence in body area networks," IEEE Vehicular Technology Conference Fall (VTC), pp.1-5, September 2010.
[4] N. Chahat, G. Valerio, M. Zhadobov and R. Sauleau, "On-body propagation at $60 \mathrm{GHz}$," IEEE Transactions on Antennas and Propagation, vol. 61, no. 4, pp. 1876-1888, April 2013.

[5] M. D. Yacoub, "The $\kappa-\mu$ and the $\eta-\mu$ distribution," IEEE Antennas Propagation Magazine, vol. 49, no. 1, pp. 68-81, Feb. 2007.

[6] S. L. Cotton, "Human Body Shadowing in cellular device-todevice communications: channel modeling using the shadowed $\kappa-\mu$ fading model," under revision, IEEE Journal on Selected Areas in Comms Special Issue on Device-to-Device Comms in Cellular Networks, 2013.

[7] J. F. Paris, "Statistical characterization of $\kappa-\mu$ shadowed fading," IEEE Transactions on Vehicular Technology, vol. 63, no. 2, pp. 518-526, February 2014.

[8] S. O. Rice, "Statistical properties of a sine wave plus random noise," Bell Syst. Tech. Journal, vol. 27, pp. 109-157, 1948.

[9] M. Nakagami, "The m-distribution: A general formula of intensity distribution of rapid fading," in Statistical Methods in Radio Wave Propagation. New York: Pergamon, 1960, pp. 336.

[10] S. L. Cotton and W. G. Scanlon, "Higher order statistics for lognormal small-scale fading in mobile radio channels," IEEE Antennas and Wireless Propagation Letters, vol. 6, pp. 540$543,2007$. 